\title{
Generation of cell-free extracts of Xenopus eggs and demembranated sperm chromatin for the assembly and isolation of in vitro-formed nuclei for Western blotting and scanning electron microscopy (SEM)
}

\author{
TD Allen ${ }^{1}$, SA Rutherford ${ }^{1}$, S Murray ${ }^{1}$, HS Sanderson ${ }^{2}$, F Gardiner $^{1}$, E Kiseleva $^{3}$, MW Goldberg ${ }^{4} \&$ SP Drummond $^{1}$ \\ ${ }^{1}$ Paterson Institute for Cancer Research, University of Manchester, Wilmslow Road, Withington, Manchester M20 4BX, UK. ${ }^{2}$ Biomedical Research Centre, University of \\ Dundee, Level 5, Ninewells Hospital and Medical School, Dundee DD1 9SY, UK. ${ }^{3}$ Institute of Cytology and Genetics, Russian Academy of Sciences, Novosibirsk-90, \\ 630090, Russia. ${ }^{4}$ School of Biological and Biomedical Sciences, Durham University, South Road, Durham DH1 3LE, UK. Correspondence should be addressed to T.D.A. \\ (tallen@picr.man.ac.uk).
}

Published online 10 May 2007; doi:10.1038/nprot.2007.138

\begin{abstract}
This protocol details methods for the generation of cell-free extracts and DNA templates from the eggs and sperm chromatin, respectively, of the clawed toad Xenopus laevis. We have used this system with scanning electron microscopy (SEM), as detailed herein, to analyze the biochemical requirements and structural pathways for the biogenesis of eukaryotic nuclear envelopes (NEs) and nuclear pore complexes (NPCs). This protocol requires access to female frogs, which are induced to lay eggs, and a male frog, which is killed for preparation of the sperm chromatin. Egg extracts should be prepared in $1 \mathrm{~d}$ and can be stored for many months at $-80^{\circ} \mathrm{C}$. Demembranated sperm chromatin should take only approximately $2-3 \mathrm{~h}$ to prepare and can be stored at $-80{ }^{\circ} \mathrm{C}$ almost indefinitely. The time required for assembly of structurally and functionally competent nuclei in vitro depends largely on the quality of the cell-free extracts and, therefore, must be determined for each extract preparation.
\end{abstract}

\section{INTRODUCTION}

Xenopus laevis has been used as a model organism for the study of vertebrate cellular structures and processes owing to the ability of cell-free extracts, generated from unfertilized eggs, to recapitulate cell-cycle events in vitro ${ }^{1,2}$. The power of biochemistry combined with the amenability of extracts to biochemical manipulation has facilitated identification and characterization of the mechanisms and proteins required for diverse events such as nuclear reassembly, nucleo-cytoplasmic trafficking, DNA replication and chromatin processing ${ }^{3-6}$.

The Xenopus cell-free system is a powerful tool for the study of cellular processes because cellular viability is not a prerequisite for the end point of such analyses. Therefore biochemical agents lethal in a cellular environment, such as non-hydrolysable forms of GTP (e.g., GTP $\gamma$ S) or the calcium chelating agent 1,2-bis(oaminophenoxy)ethane- $N, N, N^{\prime}, N^{\prime}$-tetraacetic acid, can be added and their effects on nuclear processes can be assayed ${ }^{7}$. In addition, it is possible to deplete proteins, using conventional affinity chromatography techniques, to characterize specific protein interactions and functions.

One focus of our laboratory has been the mechanisms and structural assembly pathways in nuclear envelope (NE) and nuclear pore complex (NPC) formation. To this end we have used both biochemical manipulation of egg extracts and high-resolution visualization (e.g., field emission scanning electron microscopy, or FESEM) to characterize intermediate steps in both NE and NPC assembly $^{8-11}$. In particular, we have characterized intermediate structures present on and within the NE that form nascent and mature NPCs. These studies are complemented by analyses performed in amphibian and mammalian tissue culture cells and the structural information gleaned from visualization of the giant Xenopus oocyte nucleus. Our protocols for these studies have been published previously ${ }^{12,13}$. Surface imaging, at high resolution by scanning electron microscopy (SEM), over extensive areas of membrane can identify rare or relatively transient structural morphologies that would be difficult, if not impossible, to detect using conventional thin-section transmission electron microscopy (TEM). For example, dimples, which are the very earliest intermediate structures in NPC assembly, where the inner and outer nuclear membranes fuse before subsequently dilating to form a stabilized pore, have a diameter of only $5-35 \mathrm{~nm}$ and very rapidly accumulate more complex NPC structures. Atomic force microscopy and cryo-electron microscopy have proved useful in the visualization of unfixed samples; however, results from these techniques are generated from the averaging of data sets, and therefore rare structures are again likely to be missed.

Nuclei can be assembled in cell-free extracts of Xenopus eggs and then visualized by phase and direct and indirect immunofluorescence. These techniques are useful for quality control purposes before processing samples for more labor-intensive techniques such as TEM or SEM. In addition, in vitro-assembled nuclei can be processed for SDS-polyacrylamide gel electrophoresis and Western blotting as end points in biochemical manipulation of the nuclear assembly pathway. In this protocol nuclear assembly, isolation and then processing for Western blotting and SEM are described.

\section{MATERIALS}

REAGENTS

-X. laevis eggs (see REAGENT SETUP) ! CAUTION General animal husbandry

and use must be in accordance with relevant guidelines and regulations.

\footnotetext{
One male $X$. laevis ! CAUTION General animal husbandry and use must be in accordance with relevant guidelines and regulations.

- Cysteine (Fluka Biochemika, cat. no. 30089) (see REAGENT SETUP)
} 


\section{BOX 1 | ACQUISITION AND PREPARATION OF DEMEMBRANATED SPERM CHROMATIN}

The Xenopus cell-free system has been fundamentally important in recapitulating cell-cycle events in vitro for biochemical investigation. To assemble functionally and structurally competent nuclei that can undergo complex processes such as DNA replication it is necessary to assemble the nucleus around a DNA template. Xenopus sperm chromatin is an ideal substrate as it is derived from the same organism. However, it must be processed before inclusion in egg-extract assays as the DNA template is encapsulated in a membrane 'coat' that would prevent access of extractspecific proteins. It is informative to note that upon adding sperm chromatin to extracts it undergoes extensive remodeling, with several spermspecific basic proteins being replaced by Xenopus histones $\mathrm{H} 2 \mathrm{~A}$ and $\mathrm{H} 2 \mathrm{~B}$ as the chromatin decondenses.

1. Dissect the testes ${ }^{1}$ from the male frog and rinse in solution $X$. It is important to try to remove as much attached tissue and blood as possible.

2. Macerate the testes with watch-maker's forceps to liberate the sperm into $1 \mathrm{ml}$ solution $X$ and centrifuge at $300 \mathrm{~g}$ for $10 \mathrm{~s}$ in a clinical centrifuge to remove any somatic tissue. This procedure is repeated until a maximal amount of sperm is released.

3. Centrifuge the final supernatant at $700 \mathrm{~g}$ for $2 \mathrm{~min}$ to pellet the sperm, isolate and re-suspend the cleaner, upper part of the sperm-containing pellet in $100 \mu \mathrm{l}$ solution $X$ and transfer to a clean microfuge tube.

4. Add $300 \mu$ solution $X$ containing $0.05 \%$ lysolecithin and, after gentle mixing, incubate for 5 min at $20^{\circ} \mathrm{C}$.

See ? TROUBLESHOOTING section below

5. Add 3 volumes of solution $X$ containing $3 \%$ BSA and centrifuge for 10 min at $400 \mathrm{~g}$.

6. After removing the supernatant, re-suspend the sperm in $200 \mu \mathrm{l}$ solution $\mathrm{X}$ containing $3 \% \mathrm{BSA}$ and then supplement with a further $1 \mathrm{~mL}$ of the same and pellet as in Step 5.

7. Re-suspend the final pellet in $50 \mu \mathrm{l}$ of solution $\mathrm{X}$ and determine the sperm concentration using a hemocytometer.

PAUSE POINT Store the sperm in $5-\mu \mathrm{l}$ aliquots at $-80{ }^{\circ} \mathrm{C}$ at an approximate volume of $10^{4}$ sperm per $\mu \mathrm{l}$.

See ? TROUBLESHOOTING section below

- Cyclohexamide (Sigma Aldrich, cat. no. R750107) (see REAGENT SETUP)

- Cytochalasin B (Sigma Aldrich, cat. no. C6762) (see REAGENT SETUP)

- Aprotinin and leupeptin (Sigma Aldrich, cat. nos. A6103 and L2884) (see

REAGENT SETUP)

- Lysis buffer (see REAGENT SETUP)

- Membrane wash buffer (MWB) (see REAGENT SETUP)

- Solution X (see REAGENT SETUP)

- Lysolecithin (Fluka Biochemika, cat. no. 62963)

- BSA (Sigma Aldrich, cat. no. A7030)

-Xenopus egg extracts; either crude extract (LSS, low speed supernatant) or aliquots of membranes and cytosol generated by high-speed fractionation (see Steps 1-12)

- Arklone (trichlorotrifluoroethane; ICI chemicals)

- Demembranated sperm chromatin (see Box 1)

-E-mix (containing ATP, Sigma Aldrich, cat. no. A7699; creatine phosphate,

Fluka Biochemika, cat. no. 27920; creatine kinase, Sigma Aldrich,

cat. no. C7886) (see REAGENT SETUP)

- Nuclear isolation buffer (NIB) (see REAGENT SETUP)

- Percoll (Sigma Aldrich, cat. no. P1644)

-25\% sucrose (Sigma Aldrich, cat. no. S7903)

- SDS sample buffer (see REAGENT SETUP)

- Fix buffer (see REAGENT SETUP)

- Creatine phosphate

- Sodium cacodylate

- Uranyl acetate ! CAUTION Uranyl acetate must be kept and used within a fume cabinet.

- Osmium tetroxide ! CAUTION Osmium tetroxide is toxic and must be kept and used within a fume cabinet and disposed of appropriately once used.

-Ethylene glycol bis-(succinic acid $N$-hydroxysuccinimide ester)

- Texas Red Streptavidinantibody (Amersham International)

-4',6-diamidino-2-phenylindole (DAPI)

EQUIPMENT

-A range of volume-graded glassware and measuring cylinders; number and size is dependent on the volume of starting material (eggs)

- Centrifuge capable of accepting multiples of approximately 10-ml volumes and centrifuging at $1,800 \mathrm{~g}$

- High-speed centrifuge (e.g., Sorvall) capable of centrifuging multiples of $1-2-\mathrm{ml}$ volumes at $200,000 \mathrm{~g}$
- Clinical centrifuge

$\cdot 10-15-$ and 50 -ml tubes that fit in the clinical centrifuge

-10-ml Starsted tube

- Eppendorf tubes

$\cdot 22{ }^{\circ} \mathrm{C}$ water bath

- Centrifuge accepting 5-ml samples in swing-out rotor and centrifuging at $3,000 \mathrm{~g}$

- Eppendorf bench-top microfuge

$\cdot 10$-ml disposable syringe

-21-gauge needle

- Perspex 'doughnut' reservoirs (see Fig. 1)

- Paraffin wax (stored with a fine paintbrush at approximately $60{ }^{\circ} \mathrm{C}$ )

- Glass coverslips (minimum diameter of $10 \mathrm{~mm}$ )

\section{REAGENT SETUP}

$X$. laevis eggs Freshly laid into $0.1 \mathrm{M} \mathrm{NaCl}^{14}$. $\triangle$ CRITICAL All solutions should be prepared fresh on the day of use.

Lysis buffer $10 \mathrm{mM}$ HEPES, $250 \mathrm{mM}$ sucrose, $50 \mathrm{mM} \mathrm{KCl}, 2.5 \mathrm{mM} \mathrm{KCl}$, $1 \mathrm{mM}$ DTT and $0.1 \mathrm{M} \mathrm{NaCl}$.

Cysteine A 2\% solution (in water; $\mathrm{pH} 8.0$ with $\mathrm{KOH}$ ).

Cycloheximide Inhibits protein synthesis and can be stored as a $10 \mathrm{mg} \mathrm{ml}^{-1}$ stock (in water) at $4{ }^{\circ} \mathrm{C}$.

Cytochalasin B Inhibits actin polymerization and can be stored as a

$10 \mathrm{mg} \mathrm{ml}^{-1}$ stock (in DMSO) at $-20^{\circ} \mathrm{C}$.

Aprotinin and leupeptin These protease inhibitors can be stored as a cocktail containing $1 \mathrm{mg} \mathrm{ml}^{-1}$ of each component at $-20{ }^{\circ} \mathrm{C}$.

MWB $200 \mathrm{mM}$ sucrose, $50 \mathrm{mM} \mathrm{KCl}, 2.5 \mathrm{mM} \mathrm{MgCl}_{2}, 15 \mathrm{mM}$ HEPES, pH 7.4, and $1 \mathrm{mM}$ DTT.

20× E-mix $40 \mathrm{mM}$ ATP, $200 \mathrm{mM}$ creatine phosphate, $1 \mathrm{mg} \mathrm{ml}^{-1}$ creatine kinase. Stored as a $20 \times$ stock at $-20^{\circ} \mathrm{C}$.

NIB $20 \mathrm{mM}$ Tris-HCl, pH 7.4, $70 \mathrm{mM} \mathrm{KCl}, 5 \mathrm{mM} \mathrm{MgCl}$, 2 mM DTT,

$2 \%$ polyvinylpyrolidone.

Fix buffer $80 \mathrm{mM}$ PIPES-KOH, pH 6.8, $150 \mathrm{mM}$ sucrose, $30 \mathrm{mM} \mathrm{KCl}, 1 \mathrm{mM}$ $\mathrm{MgCl}_{2}, 0.25 \%$ gluteradehyde (EM grade) and 2\% paraformaldehyde (Sigma Aldrich).

Solution X 15 mM PIPES pH 7.4, $80 \mathrm{mM} \mathrm{KCl}, 5 \mathrm{mM}$ EDTA, $15 \mathrm{mM} \mathrm{NaCl}$, $0.02 \mathrm{M}$ sucrose and $7 \mathrm{mM} \mathrm{MgCl}_{2}$.

SDS sample buffer $100 \mathrm{mM}$ Tris, pH 6.8, 2\% SDS, 5\% $\beta$-mercaptoethanol, $15 \%$ glycerol and enough bromophenol blue to make it look dark.

\section{PROCEDURE}

\section{Generation of Xenopus nuclear assembly extracts}

1| Rinse eggs in successive washes of at least 5 volumes of $0.1 \mathrm{M} \mathrm{NaCl}$ until any debris has been removed. Drain excess liquid carefully (a little can be left to ensure that eggs do not dry out and that they are not accidentally discarded). 
$\triangle$ CRITICAL STEP Pre-cool all centrifuges to $4^{\circ} \mathrm{C}$; solutions should be kept on ice before starting procedure.

2) Add at least 2-3 volumes of 2\% Cysteine and gently 'swirl' eggs; this chemically removes the thick jelly-like covering of each egg.

$\triangle$ CRITICAL STEP Gently agitate eggs intermittently for no longer than $10 \mathrm{~min}$ in total, as excessive time in the presence of Cysteine will lead to egg lysis and proteolysis.

\section{? TROUBLESHOOTING}

3| Rinse eggs three times with at least 5 volumes of $0.1 \mathrm{M} \mathrm{NaCl}$ and then three times with 3 volumes of lysis buffer. (See Fig. 2 for illustration of Steps 3-6.)

4| Remove excess buffer and add protease inhibitor cocktail (aprotonin and leupeptin; see REAGENT SETUP) to a final concentration of $3 \mu \mathrm{g} \mathrm{ml}^{-1}$ and 'swirl' to mix. Volume added will be negligible and should not be drained off after addition.

5| Immediately transfer eggs to a 10-ml Starsted tube and centrifuge for 2 min at $1,800 \mathrm{~g}$ to 'pack' eggs and then carefully remove excess buffer.

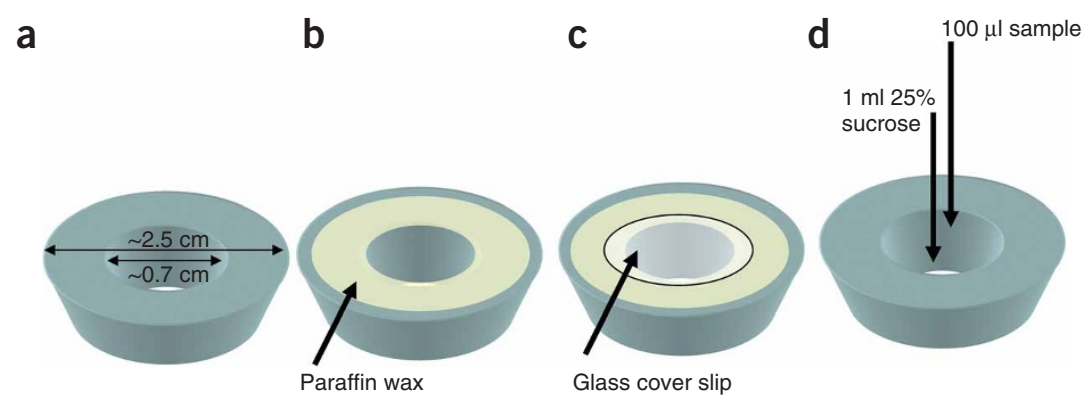

e

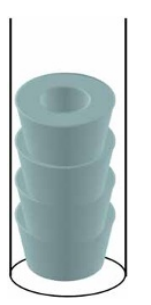

f

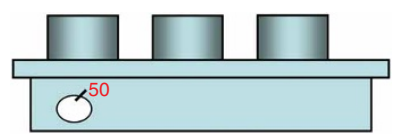

g

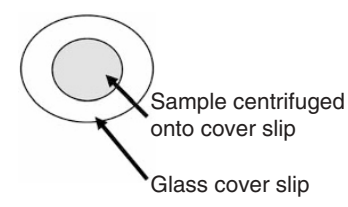

Figure 1 | Apparatus and method for centrifugation of in vitro-assembled nuclei onto glass coverslips. (a) Perspex 'doughnuts' are manufactured with an approximate outer diameter of $2.5 \mathrm{~cm}$ and an inner diameter of $0.7 \mathrm{~cm}$. The greater diameter must be such that they can fit into a $50-\mathrm{ml}$ centrifuge tube (see $\mathbf{e}$ ), and the inner diameter must be less than the diameter of the glass coverslips. (b) Use a fine paintbrush to paint the upper rim of the 'doughnut' with melted paraffin wax. (c) Gently drop a glass coverslip over the central hole of the Perspex 'doughnut' so that it adheres to the wax. (d) Invert the 'doughnut' and attached coverslip to generate a reservoir and add $1 \mathrm{ml} 25 \%$ sucrose (in nuclear isolation buffer), then carefully layer the 100- $\mu$ l sample onto the top of the sucrose cushion. (e) 'Doughnuts' are stacked in a $50-\mathrm{ml}$ centrifuge tube and centrifuged for $10 \mathrm{~min}$ at 2,000 $\mathrm{g}$. (f) After gentle pipetting to remove the residual sucrose, the coverslips are heated to $50{ }^{\circ} \mathrm{C}$ on a heated table, just until the wax begins to melt, and then the coverslips are dislodged by application of gentle pressure with forceps through the center of the 'doughnut'. (g) This leaves a coverslip with the sample centrifuged on to the center. (Note that for clarity in the diagram the outer sides of the doughnuts are drawn with a tapered outer diameter, although they are actually made by cutting a hollow rod of Perspex.)

6| Lyse eggs by centrifuging at $10,000 \mathrm{~g}$ for $12 \min$ at $4^{\circ} \mathrm{C}$.

7| Recover and isolate the crude extract with a $10-\mathrm{ml}$ syringe and 21-gauge needle through the wall of the centrifuge tube, taking care to minimize contamination with yolk and pigment layers.

8| Transfer to a clean tube and supplement with further protease inhibitor cocktail to a final concentration of $3 \mu \mathrm{gl}^{-1}$. $\triangle$ CRITICAL STEP The crude extract collected at this stage, also termed LSS, can be used for nuclear assembly studies, although often it is preferable to fractionate it into its cytosolic and membrane-containing fractions (see Steps 9-12 below) for subsequent biochemical analysis.

PAUSE POINT Store in $20-\mu \mathrm{l}$ aliquots at $-80^{\circ} \mathrm{C}$ or under liquid nitrogen.

a

b

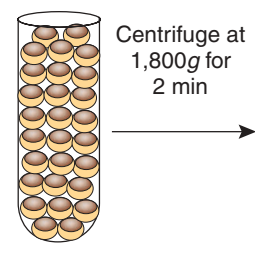

Remove excess buffer from top of tube
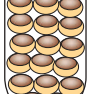

C

d
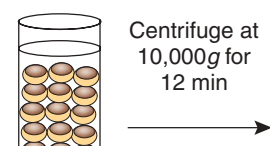

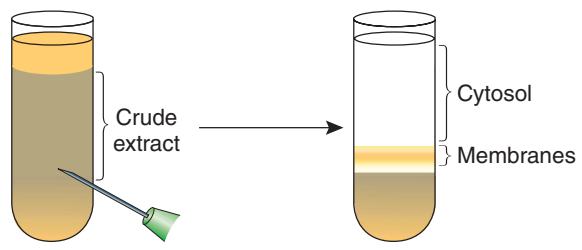

Crude extract removed using syringe and needle
Figure 2 | Xenopus nuclear assembly extracts. (a) Eggs from Xenopus laevis have the surrounding jelly coat removed and are then transferred into a centrifuge tube and packed. (b) Excess buffer is removed from the top of the centrifuge tube. (c) Eggs are fractionated to yield a crude extract, which is then isolated and subjected to a high-speed spin to generate (d) membrane and cytosolic fractions.
9| Centrifuge crude extract at $200,000 \mathrm{~g}$ for $1 \mathrm{~h}$ at $4{ }^{\circ} \mathrm{C}$ in an ultracentrifuge. For the most part these centrifuges accept tubes up to $2 \mathrm{ml}$ in volume.

10| Transfer the cytosolic fraction, which is the clear membrane-free aqueous phase as indicated in Figure 2, to a clean ultracentrifuge tube and retain the membrane fraction on ice.

11 Centrifuge the cytosolic fraction for a further $15 \mathrm{~min}$ at $200,000 \mathrm{~g}$ at $4{ }^{\circ} \mathrm{C}$ to remove residual membranes. 
PAUSE POINT Snap-freeze the cytosol in liquid nitrogen in $20-, 50-$ or $100-\mu l$ aliquots depending on volumes yielded and projected experimental use.

12| Transfer the membrane fraction (generated at Step 10) to a clean tube on ice, re-suspend in MWB and pellet by centrifugation at $30,000 \mathrm{~g}$ for $15 \mathrm{~min}$ at $4^{\circ} \mathrm{C}$.

- PAUSE POINT Remove as much excess buffer as possible and freeze membranes in 10-20- $\mu$ l aliquots in liquid nitrogen and store as described for cytosol.

\section{In vitro nuclear assembly and isolation}

13| To initiate nuclear formation, assemble reactions containing either LSS (from Step 8) or membranes re-suspended in cytosol in the same proportions as yielded (often approximately 1:10 membranes:cytosol) in Eppendorf tubes.

14 Add E-mix to a final concentration of $1 \times$ and mix gently but thoroughly.

\section{? TROUBLESHOOTING}

15 Add Xenopus demembranated sperm (see Box 1 for preparation) to a final concentration of 1,000 sperm per $\mu$ l and incubate at $22{ }^{\circ} \mathrm{C}$ for 90 min.

$\triangle$ CRITICAL STEP Every extract will vary in the efficiency of nuclear assembly, and therefore it is necessary initially to monitor nuclear assembly to determine optimal incubation times for each extract generated. Assembly can be assayed using visual and biochemical markers, such as observation by conventional phase microscopy or the incorporation of suitably labeled dUTPs indicating DNA replication competency. Assembled nuclei must be processed immediately for SEM or suspended in SDS sample buffer for Western blotting.

\section{? TROUBLESHOOTING}

16| After assaying the efficiency of nuclear assembly (Box 2), isolate and process assembled nuclei for Western blotting (option A) or high-resolution SEM visualization (option B).

\section{(A) Nuclear isolation for Western blotting}

(i) Dilute $50-\mu \mathrm{l}$ aliquots of assembly reaction (from Step 15) in $1 \mathrm{ml}$ ice-cold NIB in a 1.5-ml microfuge tube. Typically $2 \times 10^{5}$ nuclei are sufficient for loading into a single $30-\mu \mathrm{l}$ acylamide gel well; however, samples may need to be generated in excess to ensure ease of manipulation and adequate efficiency of nuclear isolation.

(ii) Pool diluted aliquots and layer over at least $4 \mathrm{ml} 60 \%$ Percoll (in NIB) in a 10-ml centrifuge tube and centrifuge at 3,000g for 10 min at $4^{\circ} \mathrm{C}$.

\section{BOX 2 | IMMUNOFLUORESCENCE ASSAY FOR DNA REPLICATION COMPETENCY}

The ability and efficiency of egg extracts to support the assembly of nuclei capable of undergoing DNA replication can be analyzed by immunofluorescence using thymidine analogs such as biotin-11-dUTP (biotinylated deoxyuridine triphosphate). This procedure for determining the efficacy of nuclear assembly is quick and sensitive and directly identifies sites of DNA replication. It is advisable to use this in concert with conventional phase microscopy.

1. Reconstitute egg extracts by adding membrane and cytosolic fractions and an energy regeneration system (E-mix; see PROCEDURE Step 14) to a final volume of $50 \mu \mathrm{l}$ and add $40 \mu \mathrm{M}$ biotin-11-dUTP. Finally, add approximately $1 \times 10^{3}$ sperm per $\mu \mathrm{l}$.

2. Gently mix reagent with a wide bore pipette tip, ensuring that DNA is not sheared, and incubate at room temperature.

3. At $15-30$ min intervals remove $5 \mu$ and fix in $100 \mu \mathrm{l}$ of one-third strength extraction buffer $(110 \mathrm{mM} \mathrm{KCl}, 5 \mathrm{mM} \mathrm{MgCl}, 20 \mathrm{mM} \mathrm{HEPES}, \mathrm{pH} 7.5$,

$2 \mathrm{mM} \beta$-mercaptoethanol) containing $1 \mathrm{mM}$ ethylene glycol bis-(succinic acid $\mathrm{N}$-hydroxysuccinimide ester) at $37^{\circ} \mathrm{C}$ for $30 \mathrm{~min}$.

4. (For the following four steps, see Fig. 1.) Stick glass coverslips to Perspex 'doughnuts' using a small amount of paraffin wax and leave at room temperature for a minimum of 2 min for the wax to set.

5. Invert the Perspex 'doughnut' with attached coverslip to make a small reservoir for adding $1 \mathrm{ml} 25 \%$ sucrose (in nuclear isolation buffer).

6. Gently pipette the sample onto the top of the sucrose cushion and centrifuge at a maximum force of 2,000 $\mathrm{g}$ for $10 \mathrm{~min}$ at room temperature. Once the sample has been centrifuged, gently pipette off the remaining sucrose.

7. Heat the 'doughnuts' and attached coverslips to $50{ }^{\circ} \mathrm{C}$ (coverslip side nearest the heat source) for just long enough for the wax to begin to melt so that the coverslip can be removed by gently pressing with forceps through the center of the 'doughnut'.

8. Wash coverslips in PBS. Coverslips can be manipulated manually using forceps and lowered into PBS in a minimum of three beakers.

9. Incubate coverslips with Texas Red streptavidin antibody (Amersham International; for times and concentrations follow manufacturer's guidelines).

10. Wash thoroughly with PBS and mount slides in 50\% glycerol containing $1 \mu \mathrm{g} \mathrm{ml}^{-1} 4^{\prime}, 6$-diamidino-2-phenylindole (DAPI) (to identify DNA). 11. View nuclei with a fluorescence microscope fitted with a UV source.

With this method, nuclei actively undergoing DNA replication are clearly seen with a strong red signal co-localizing with DNA (as identified with DAPI). This method also allows for direct observation of nuclear morphology and ideally fully formed nuclei should be relatively spherical with evenly decondensed chromatin. Typical nuclear diameter is approximately $10 \mu \mathrm{m}$. A timecourse of assembly and assaying for DNA replication ability should be performed to determine when, and if, these criteria are met. 
(iii) Collect material from the NIB/Percoll interface in a 10-ml centrifuge tube and dilute to $1 \mathrm{ml}$ with ice-cold NIB.

(iv) Layer the diluted nuclei over $0.5 \mathrm{ml} 25 \%$ sucrose (wt/vol in NIB) in a 1.5-ml Eppendorf tube and centrifuge in an Eppendorf 5810R bench-top microfuge at 2,500 r.p.m. to yield a nuclear pellet.

(v) Re-suspend the nuclear pellet in SDS sample buffer for loading of approximately $2 \times 10^{5}$ nuclei per gel lane.

(vi) Perform SDS-PAGE and Western blotting according to standard procedures.

\section{(B) Nuclear processing for visualization by SEM}

(i) Dilute a 4- $\mu$ l aliquot of each assembly reaction (from Step 15) and dilute in $500 \mu \mathrm{l} \mathrm{MWB.}$

(ii) Transfer diluted sample into clean flat-based tube holding a silicon chip at the bottom and sediment nuclei onto acetonewashed silicon chips by centrifugation at $3,000 \mathrm{~g}$ for 10 min at $4{ }^{\circ} \mathrm{C}$.

(iii) Transfer chips and incubate for 10 min in fix buffer, ensuring a minimum of MWB dilutes the fixative.

(iv) Remove fix buffer while ensure that chips do not air-dry and wash chips in $0.2 \mathrm{M}$ sodium cacodylate ( $\mathrm{pH} 7.2$ in water) to remove residual fix buffer.

(v) Post-fix samples in 1\% osmium tetroxide (in $0.2 \mathrm{M}$ sodium cacodylate, $\mathrm{pH} 7.2$ ) for 10-20 min. Please note that osmium preserves lipid and phospholipid content and also enhances contrast in the specimen.

(vi) Remove osmium tetroxide and wash in water for $10 \mathrm{~s}$

(vii) Remove water and stain for 10 min in $1 \%$ uranyl acetate (in water).

(viii) Dehydrate through a graded ethanol series (30,50, 70, 95 and twice at 100\%) and twice in $100 \%$ Arklone (trichlorotrifluoroethane; ICI) or other suitable transitional solvent.

(ix) Critical-point-dry the samples from high purity (less than 5 p.p.m. water) $\mathrm{CO}_{2}$. (More information on critical point drying is available at http://www.emitech.co.uk/cpd-brief.htm.)

(x) Sputter-coat the samples with 2-3 nm chromium. (More information on chromium coating of samples for FESEM is available at http://www.quorumtech.com/Manuals/Current_Technical_Briefs/TB-SPUTTER.pdf.)

(xi) Image the samples using FESEM.

\section{TIMING}

Female Xenopus must first be primed (injected with pregnant mare's serum gonadotrophin 2-7 $\mathrm{d}$ before the induction of egg laying); ovulation is then induced by injection of human chorionic gonadotrophin the day before eggs are required. Eggs are collected approximately $16 \mathrm{~h}$ after the final injection ${ }^{14}$.

All solutions can be prepared before embarking on the procedure but should be prepared on the day of use.

Steps 1-12, generation of Xenopus nuclear assembly extracts: approximately $2.5 \mathrm{~h}$

Steps 13-15, in vitro nuclear assembly and isolation assembly reactions should be reconstituted as quickly as is feasible, always adding the demembranated chromatin last. Full nuclear assembly is likely to be supported between 90 and 180 min

Step 16A, nuclear isolation for western blotting: approximately 40 min

Step 16B, nuclear processing for visualization by SEM: approximately $1 \mathrm{~h} 10 \mathrm{~min}$, plus the time required for sputter-coating using the coater available

Acquisition and preparation of demembranated sperm chromatin (Box 1): approximately $1.5 \mathrm{~h}$

Immunofluorescence assay for DNA replication competency (Box 2): approximately $3 \mathrm{~h}$

\section{? TROUBLESHOOTING}

Troubleshooting advice can be found in Table 1.

TABLE 1 | Troubleshooting table.

\begin{tabular}{|c|c|c|c|}
\hline Step & Problem & Possible reason & Solution \\
\hline $\begin{array}{l}\text { Before initiation of } \\
\text { procedure and Steps } \\
1 \text { and } 2\end{array}$ & $\begin{array}{l}\text { Very inefficient or incomplete/arrested } \\
\text { assembly as evidenced by incomplete } \\
\text { decondensation of chromatin substrate, } \\
\text { incomplete membrane binding and/or }\end{array}$ & Poor quality extract & $\begin{array}{l}\text { Check the health of your frogs and } \\
\text { remember to remove any opaque/white } \\
\text { eggs before making extract both before } \\
\text { and after the de-jellying stage }\end{array}$ \\
\hline
\end{tabular}

Step 4 in Box 1
Incomplete sperm demembranation
Test for the presence of membrane on sperm preparation by staining with a nonspecific lipid dye such as diheyxloxacarbocyanine or BoDipy. If membranes are still present sperm can be re-treated with lysolecithin, although ideally the sperm should be prepared again and checked for residual membranes before aliquoting 
PROTOCOL

TABLE 1 | Troubleshooting table (continued).

\begin{tabular}{|c|c|c|c|}
\hline Step & Problem & Possible reason & Solution \\
\hline Step 14 & & E-mix has gone off & $\begin{array}{l}\text { Check expiry date of all components of } \\
\text { E-mix and avoid repeated freeze-thaw } \\
\text { cycles }\end{array}$ \\
\hline
\end{tabular}

Step 15

Wrong temperature and oxygenation during assembly reaction

Step 7 in Box 1

Too high a sperm concentration

Wrong pipette tip size used

All steps once sperm Broken nuclei and sheared DNA
Nuclear assembly reactions should be performed at approximately $22{ }^{\circ} \mathrm{C}$ as temperatures lower than this will delay and potentially arrest assembly, and higher temperatures will denature proteins and assembly will not proceed correctly

Check sperm stock concentration using a haemocytometer and then perform a titration experiment to determine optimal concentration for extract has been added to reaction
Avoid vigorous pipetting of extract once sperm has been added and do not use small-volume tips; if necessary cut off the end of the tip
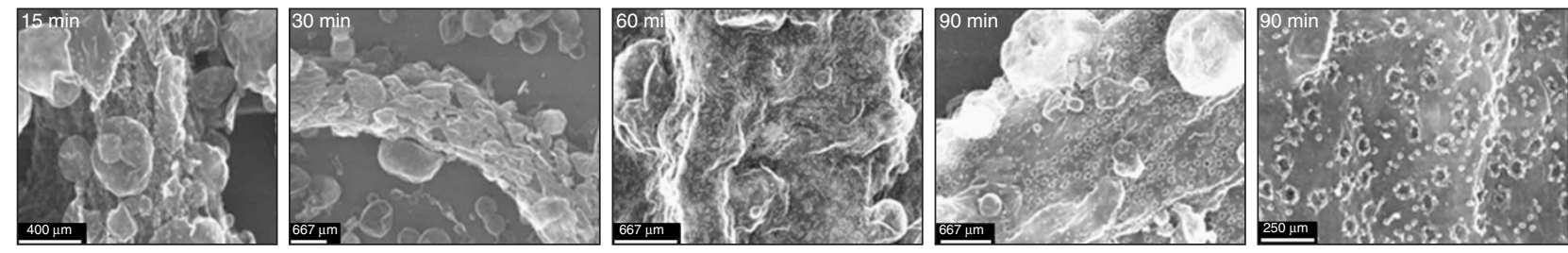

Figure 3 | Visualization of in vitro nuclear assembly by field emission scanning electron microscopy (FESEM). Nuclei were assembled in vitro for 90 min. Samples of the assembly reaction were taken at the indicated time points and then processed and viewed by FESEM. Representative images detailing the progression of nuclear envelope (NE) assembly and the timing of nuclear pore complex (NPC) insertion are shown. There is binding of vesicles to chromatin (15 min) and fusion of vesicles with one another to enclose the de-condensing chromatin (30 min). These vesicles then flatten to the chromatin surface and the chromatin is enclosed by a wrinkled NE (60 min), which then becomes smoother (90 min), presumably owing to an increase in nuclear volume and possibly establishment of associations with components of the NE and underlying nuclear structures. The typical morphology and distribution of mature NPCs are shown in both images captured at the 90 min time point.

\section{ANTICIPATED RESULTS}

Critical factors for the generation of high-quality cell-free egg extracts include ensuring that the frogs from which the eggs are collected are maintained at the highest level of health and are as stress-free as possible. It is also important to assemble all buffers on the day of use, and where possible maintain them at $4{ }^{\circ} \mathrm{C}$ or on ice. The average yield of crude extract is approximately half the starting volume of eggs. If this is further fractionated into membrane and soluble components, they will be generated in relative proportions of approximately 1:10, respectively. It is important to determine optimal volumes for reconstitution of each extract used for nuclear assembly reactions and appropriate timescales required for the assembly as the efficiency will vary between extracts. Figure 3 shows FESEM visualization of characteristic structural events in the assembly of a nucleus in cell-free extracts of Xenopus eggs.

COMPETING INTERESTS STATEMENT The authors declare no competing financial interests.

Published online at http://www.natureprotocols.com

Reprints and permissions information is available online at http://npg.nature.com/ reprintsandpermissions

1. Lohka, M.J. \& Masui, Y. Formation in vitro of sperm pronuclei and mitotic chromosomes induced by amphibian ooplasmic components. Science $\mathbf{2 2 0}$, 719-721 (1983).
2. Lohka, M.J. \& Maller, J.L. Induction of nuclear envelope breakdown, chromosome condensation, and spindle formation in cell-free extracts. J. Cell Biol. 101, 518-523 (1985).

3. Chan, R.C. \& Forbes, D.I. In vitro study of nuclear assembly and nuclear import using Xenopus egg extracts. Methods Mol. Biol. 322, 289-300 (2006).

4. Smythe, C. \& Newport, J.W. Systems for the study of nuclear assembly, DNA replication, and nuclear breakdown in Xenopus laevis egg extracts. Methods Cell Biol. 35, 449-468 (1991).

5. Tutter, A.V. \& Walter, J.C. Chromosomal DNA replication in a soluble cell-free system derived from Xenopus eggs. Methods Mol. Biol. 322, 121-137 (2006). 
6. Maresca, T.J. \& Heald, R. Methods for studying spindle assembly and chromosome condensation in Xenopus egg extracts. Methods Mol. Biol. 322, 459-474 (2006).

7. Macaulay, C. \& Forbes, D.J. Assembly of the nuclear pore: biochemically distinct steps revealed with NEM, GTP gamma S, and BAPTA. J. Cell Biol. 132, 5-20 (1996).

8. Goldberg, M.W., Wiese, C., Allen, T.D. \& Wilson, K.L. Dimples, pores, star-rings, and thin rings on growing nuclear envelopes: evidence for structural intermediates in nuclear pore complex assembly. J. Cell Sci. 110, 409-420 (1997).

9. Wiese, C., Goldberg, M.W., Allen, T.D. \& Wilson, K.L. Nuclear envelope assembly in Xenopus extracts visualized by scanning EM reveals a transport-dependent 'envelope smoothing' event. J. Cell Sci. 110, 1489-1502 (1997).
10. Drummond, S.P. \& Wilson, K.L. Interference with the cytoplasmic tail of gp210 disrupts 'close apposition' of nuclear membranes and blocks nuclear pore dilation. J. Cell Biol. 158, 53-62 (2002).

11. Walther, T.C. et al. The conserved Nup107-160 complex is critical for nuclear pore complex assembly. Cell 113, 195-206 (2003).

12. Allen, T.D. et al. A protocol for isolating Xenopus oocyte nuclear envelope for visualization and characterization by scanning electron microscopy (SEM) or transmission electron microscopy (TEM). Nat. Protoc. 2, 1166-1172 (2007).

13. Allen, T.D. et al. Visualization of the nucleus and nuclear envelope in situ by SEM in tissue culture cells. Nat. Protoc. 2, 1180-1184 (2007).

14. Lohka, M.J. Analysis of nuclear envelope assembly using extracts of Xenopus eggs. Methods Cell Biol. 53, 367-395 (1998). 